\title{
Porto-Pulmonary Hypertension (POPH) in a Female with Non-Cirrhotic Portal Hypertension - Early Suspicion and Early Diagnosis is the Key
}

\author{
Shivika Malik ${ }^{1}$, Abhijit Wadekar², Sourya Acharya ${ }^{3}$, Samarth Shukla, Sunil Kumar ${ }^{5}$ \\ 1, 2, 3, 5 Department of Medicine, Datta Meghe Institute of Medical Sciences (Deemed to Be University), \\ Sawangi, Wardha, Maharashtra, India. ${ }^{4}$ Department of Pathology, \\ Datta Meghe Institute of Medical Sciences (Deemed to Be University), Sawangi, Wardha, Maharashtra, India.
}

\section{INTRODUCTION}

Liver disease and portal venous disease can present with a number of pulmonary complications, such as hepatic hydrothorax, hepatopulmonary syndrome and Portopulmonary hypertension (POPH). Porto-pulmonary hypertension is a rare but serious condition. Increased vascular pressure occurs due to progressive vasoconstriction, causing respiratory discomfort to the patient and ultimately progressing to right heart failure. Porto-pulmonary hypertension falls into World Health Organization (WHO) group 1 of pulmonary hypertension, indicating that the mechanism involved in the development of the disease is pulmonary vascular remodelling. It is the rarest of all pulmonary complications of liver disease, but perhaps the most destructive, as it is impossible to reverse the changes at the vascular level. Early diagnosis is essential to decrease mortality, and investigations, such as echocardiography and right heart catheterization are available. As POPH is uncommon, not much is known about its treatment. The best therapy is proper and timely management of portal hypertension, to prevent its occurrence altogether. We present a case of a 36-year-old female, who presented with features typical of portal hypertension, but on further examination, patient was found to have pulmonary hypertension as well. Imaging findings suggested non cirrhotic portal fibrosis to have caused portal hypertension, and eventually pulmonary hypertension.

\section{PRESENTATION OF CASE}

A 36-year-old female presented to the outpatient department (OPD), with complaints of progressive exertional dyspnoea and orthopnea for the last three months. She also had one episode of hematemesis three months back and two episodes of melena in the last two months.

On examination, vitals were stable and she appeared pale. Abdominal examination revealed grade 2 splenomegaly and tender hepatomegaly, with no signs of free fluid. Jugular venous pressure (JVP) was raised and on auscultation, loud P2 was appreciated in the pulmonary area, along with high pitched early diastolic murmur at left $2^{\text {nd }}$ intercostal space and pan systolic murmur at the left lower sternal border. Laboratory investigations showed pancytopenia with a deranged coagulation profile. Since the patient had presented with hematemesis, an upper gastrointestinal (GI) endoscopy was performed, confirming the presence of oesophageal varices, which pointed towards a diagnosis of portal hypertension when correlated with the findings of abdominal examination.

Ultrasonography (USG) of abdomen showed an enlarged liver with normal echotexture, hypodense filling defect in the portal vein suggestive of a thrombus and formation of portal cavernoma. Contrast enhanced computed tomography (CECT) of the abdomen confirmed the USG findings (Figure - 1). 2D ECHO was done to assess cardiac function and pulmonary hypertension was identified by the presence of a dilated right atrium and ventricle, tricuspid regurgitation, and pulmonary arterial pressures more than $50 \mathrm{mmHg}$.
Corresponding Author:

Dr. Sourya Acharya,

Professor and Head,

Department of Medicine,

Datta Meghe Institute of Medical Sciences

(Deemed to be University), Sawangi,

Wardha, Maharashtra, India.

E-mail: Souryaacharya74@gmail.com

DOI: $10.14260 / j e m d s / 2021 / 649$

How to Cite This Article:

Malik S, Wadekar A, Acharya S, et al. Portopulmonary hypertension (POPH) in a female with non-cirrhotic portal hypertension - early suspicion and early diagnosis is the key. J Evolution Med Dent Sci 2021;10(36):3196-3198, DOI: $10.14260 /$ jemds $/ 2021 / 649$

Submission 13-05-2021,

Peer Review 30-07-2021,

Acceptance 06-08-2021,

Published 06-09-2021.

Copyright (c) 2021 Shivika Malik et al. This is an open access article distributed under Creative Commons Attribution License [Attribution 4.0 International (CC BY 4.0)] 


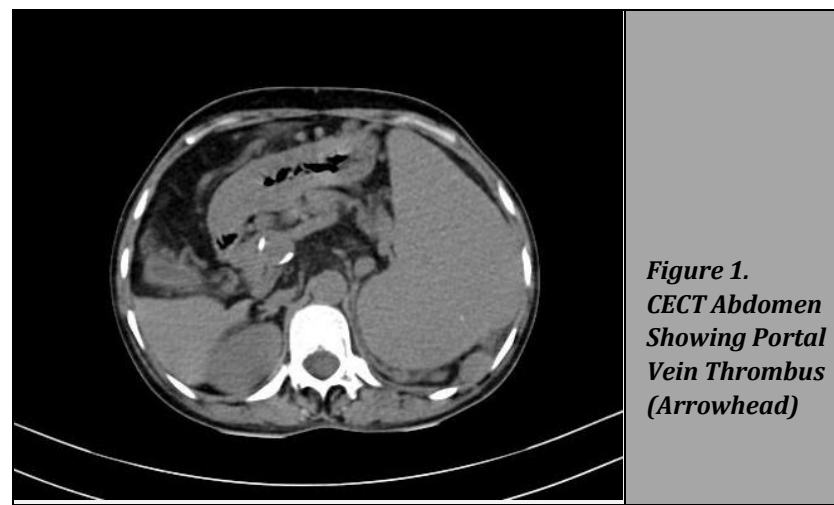

On the basis of clinical findings such as hematemesis, melena and splenomegaly, and imaging findings like varices, right heart enlargement and raised pulmonary arterial pressure, a diagnosis of Porto-pulmonary hypertension was made. The presence of portal cavernoma and portal thrombosis was suggestive of non-cirrhotic portal fibrosis, which may have been the cause of portal hypertension.

The patient was started on bosentan and sildenafil for pulmonary hypertension. Endoscopic band ligation was performed for the oesophageal varices.

\section{DISCUSSION}

Porto-pulmonary hypertension is a complication of portosystemic shunting, which may or may not be due to an underlying liver disease. It has two components - portal hypertension and pulmonary hypertension. Portal hypertension is often a clinical diagnosis, identified by the presence of splenomegaly, ascites, thrombocytopenia, and oesophageal varices. ${ }^{1} \mathrm{~A}$ portal venous pressure of more than $12 \mathrm{mmHg}$ is confirmatory.

The pathogenesis of pulmonary hypertension in the setting of portal hypertension is not completely understood, however, histological examination of pulmonary vessels in POPH has shown a similarity to pulmonary arterial hypertension, ${ }^{2}$ and hence is classified as WHO Group 1 for pulmonary hypertension. ${ }^{3}$

Portal hypertension is a hyperdynamic state, producing immense shear stress at the level of pulmonary vasculature irrespective of aetiology. Even hematologic disease can produce portal hypertension and subsequent complication. ${ }^{4}$ Extensive remodelling of the vessels occurs in response to this stress, characterised by smooth muscle hypertrophy, intimal proliferation and fibrosis, creating a pro thrombotic state and ultimately, obstruction of vessels. ${ }^{5}$ Additionally, the presence of Porto-systemic shunts allows vasoconstrictors such as endothelin-1, thromboxane A2 and vasoactive intestinal peptide (VIP) to bypass liver metabolism, creating a vasodilator-vasoconstrictor imbalance further contributing to vasoconstriction and increased pressures. ${ }^{1}$

$\mathrm{POPH}$ is a rare condition, seen in $2-6 \%$ of patients of portal hypertension. ${ }^{1}$ About $5 \%$ of the cases of pulmonary arterial hypertension are diagnosed as having POPH. ${ }^{2}$ Female sex and autoimmune liver disease predispose to development of $\mathrm{POPH},{ }^{3}$ while chronic hepatitis C infection is protective. ${ }^{2}$ The severity of disease is directly proportional to the size of Portosystemic shunts. ${ }^{2}$
Patients with POPH in the early stages present with nonspecific fatigue, ${ }^{6}$ along with features of portal hypertension like variceal bleeding and splenomegaly. In advanced disease, dyspnoea with orthopnea is the key presentation, and must be distinguished from another complication of liver disease, i.e. Hepato-pulmonary syndrome (HPS). Though clinically similar, HPS, in contrast to $\mathrm{POPH}$, is mediated by intrapulmonary vascular dilatation and can be identified by the presence of hypoxemia and platypnea. ${ }^{5}$ Long standing pulmonary hypertension generally proceeds to right heart failure, suggested by raised JVP, oedema and ascites.

The diagnosis of POPH is an easy task, provided clinical suspicion is present for the same. Trans thoracic echocardiography (TTE) is the preferred screening modality. ${ }^{7}$ Right atrial pressure can be measured indirectly, using the right ventricular systolic pressure (RVSP) and peak tricuspid regurgitant jet velocity. TTE may underdiagnose the presence of pulmonary hypertension in the absence of a tricuspid jet, ${ }^{5}$ and thus, the gold standard investigation for the diagnosis of pulmonary hypertension remains right heart catheterisation. Elevated mean pulmonary artery pressure (mPAP > $25 \mathrm{mmHg}$ at rest), or elevated transpulmonary gradient ( $>12 \mathrm{mmHg}$ ) confirms the diagnosis of pulmonary hypertension. ${ }^{3}$

The aim of treatment in POPH is to provide symptomatic relief and improve quality of life. Vasodilator therapy remains the mainstay treatment, and commonly used agents include prostanoids, like epoprostenol, endothelin-1 receptor antagonists, like bosentan and phosphodiesterase type 5 inhibitors such as sildenafil. ${ }^{8}$ Calcium channel blockers, which are the primary treatment of pulmonary hypertension are to be avoided in $\mathrm{POPH}$, to prevent worsening of portal hypertension by mesenteric vasodilatation. ${ }^{2}$ Beta blockers used to prevent variceal bleeding in portal hypertension, have a controversial role in $\mathrm{POPH}$ as they impair the exercise capacity of patients with POPH. ${ }^{5}$ Liver transplantation is associated with high mortality rate and thus, avoided. ${ }^{2}$

\section{CONCLUSIONS}

Any patient with portal hypertension and breathlessness should be evaluated by TTE to rule out POPH. Though a catastrophic progressive condition, early diagnosis paves way for early management.

Financial or other competing interests: None.

Disclosure forms provided by the authors are available with the full text of this article at jemds.com.

\section{REFERENCES}

[1] Giusca S, Jinga M, Jurcut C, et al. Portopulmonary hypertension: from diagnosis to treatment. Eur J Med 2011;22(5):441-7.

[2] Porres-Aguilar M, Mukherjee D. Portopulmonary hypertension: an update. Respirology 2015;20(2):23542.

[3] Troy PJ, Waxman AB. Portopulmonary hypertension: challenges in diagnosis and management. Therap Adv Gastroenterol 2009;2(5):281-6. 
[4] Kumar S, Joshi R, Jain AP. Portal hypertension associated with sickle cell disease. Indian J Gastroenterol 2007;26(2):94.

[5] Lee WS, Wong SY, Ivy DD, et al. Hepatopulmonary syndrome and portopulmonary hypertension in children: recent advances in diagnosis and management. The Journal of Pediatrics 2018;196:14-21.
[6] Bartolome S. Portopulmonary hypertension: diagnosis, clinical features, and medical therapy. Clin Liver Dis 2014;4(2):42-5.

[7] Porres-Aguilar M, Altamirano JT, Torre-Delgadillo A, et al. Portopulmonary hypertension and hepatopulmonary syndrome: a clinician-oriented overview. Eur Respir Rev 2012;21(125):223-33.

[8] Saleemi S. Portopulmonary hypertension. Ann Thorac Med 2010;5(1):5-9. 